\title{
Effect of orifice location on heat transfer in a duct filled with pressurized Hell
}

\section{AUTHOR(S):}

Konishi, S; Fujita, K; Okamura, T; Shirai, Y; Shiotsu, M

\section{CITATION:}

Konishi, S ... [et al]. Effect of orifice location on heat transfer in a duct filled with pressurized Hell. IEEE TRANSACTIONS ON APPLIED SUPERCONDUCTIVITY 2004, 14(2): 1762-1765

\section{ISSUE DATE:}

2004-06

URL:

http://hdl.handle.net/2433/50264

\section{RIGHT:}

(c)2004 IEEE. Personal use of this material is permitted. However, permission to reprint/republish this material for advertising or promotional purposes or for creating new collective works for resale or redistribution to servers or lists, or to reuse any copyrighted component of this work in other works must be obtained from the IEEE. 


\title{
Effect of Orifice Location on Heat Transfer in a Duct Filled With Pressurized He II
}

\author{
Soujirou Konishi, Kenji Fujita, Takahiro Okamura, Yasuyuki Shirai, and Masahiro Shiotsu
}

\begin{abstract}
Steady-state and transient heat transfer on a flat plate at one end of a rectangular duct with an orifice were measured in He II for bath temperatures of $1.8 \mathrm{~K}$ to $2.14 \mathrm{~K}$ at atmospheric pressure. The steady-state critical heat flux (CHF) was slightly affected by the orifice location. The transient heat transfer for a stepwise heat input with a magnitude larger than the CHF showed a quasi steady-state with a certain lifetime on the extrapolation of steady-state Kapitza conductance curve. The relation between the lifetime and step heat flux was significantly affected by the orifice location. Numerical analysis was performed for the steady-state and the transient heat transfer in the duct with the orifice by using the two-dimensional computer code named SUPER-2D based on the two-fluid model and the theory of mutual friction. The solutions of $\mathrm{CHF}$ and lifetime agreed well with the experimental data. The heat transport mechanism was clarified by the analysis.
\end{abstract}

Index Terms-He II, heat transfer, two-dimensional, two-fluid model.

\section{INTRODUCTION}

$\mathbf{P}$ RESSURIZED He II has excellent cooling properties in comparison with He I. Therefore, He II is expected to be a better coolant for large-scale superconducting magnets. The cooling paths of the practical superconducting magnets are very complicated. When we consider cooling of magnets, it is important to clarify heat flow in a duct with a complicated orifice like so as to resolve the instability of the magnets.

The heat transfer in He II has been mainly investigated experimentally and numerically for a one-dimensional heat flow system. Pfotenhauer [1] treated the heat flow in the path as a series of parallel connection of the thermal resistance, each due to a Gorter-Mellink duct. On the other hand, there have been a quite small number of studies on the numerical analysis for the two-dimensional transient heat transfer in He II by using the two-fluid model with some assumption. Whether the numerical code is adequate or not has not been clarified. Tatsumoto et al. [2] developed the two-dimensional numerical code "SUPER-2D" based on the two-fluid model and the theory of the mutual friction. The adequacy of the code has been confirmed by comparison with the experimental data [2], [3].

Manuscript received October 21, 2003. This research was performed as a LHD joint research project of NIFS, and was supported in part by the Japan Society for the Promotion of Science, Grant in Aid for Scientific Research (A), 12305016, 2000-2002.

S. Konishi, K. Fujita, T. Okamura, and M. Shiotsu are with Department of Energy Science and Technology, Kyoto University, Uji, Kyoto 611-0011, Japan (e-mail: konishi@pe.energy.kyoto-u.ac.jp; okamura@pe.energy.kyoto-u.ac.jp; shiotsu@pe.energy.kyoto-u.ac.jp).

Y. Shirai is with Department of Energy Science and Technology, Kyoto University, Yoshida, Kyoto 606-8501, Japan (e-mail: shirai@ pe.energy.kyoto-u.ac.jp).

Digital Object Identifier 10.1109/TASC.2004.831071

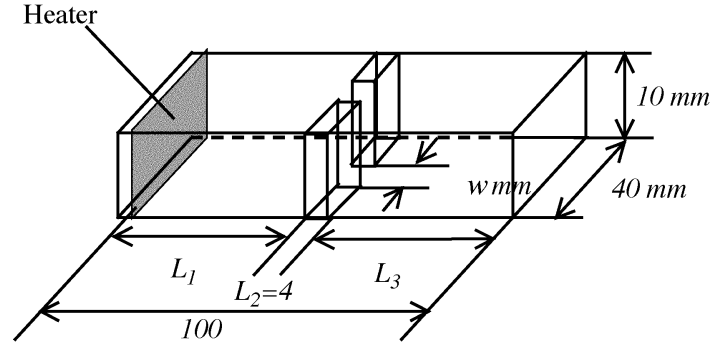

Fig. 1. Schematic of a rectangular duct with an orifice.

In this paper, steady-state and transient heat transfer in a rectangular duct with an orifice filled with He II was investigated both experimentally and numerically.

\section{EXPERIMENT}

The schematic of a rectangular duct with an orifice is shown in Fig. 1. Each flat plate heater made of Manganin was $40 \mathrm{~mm}$ in width, $10 \mathrm{~mm}$ in length and $0.1 \mathrm{~mm}$ in thickness. It was located at one end of the horizontally supported duct. The other end of the duct was opened to a He II bath. As is shown in Fig. 1, this rectangular duct can be divided into three parts; the first duct with the length of $L_{1}$ facing to the flat plate heater, an "orifice region" with the length of $L_{2}(4 \mathrm{~mm})$ and the width of w, and the second duct with the length of $L_{3}$ opened to a He II bath. Both the first and second duct have the cross sectional area $A_{d}$ (40 $\mathrm{mm} \times 10 \mathrm{~mm})$. The "orifice open mouth region" was $A_{o}$ (w $\mathrm{mm} \times 10 \mathrm{~mm}$ ) in area. Now let us define the ratio of $A_{o} / A_{d}$ (\%) as "Orifice Open Mouth."

Experiments were performed for a Orifice Open Mouth of $50 \%$ and $9 \%$, for the $L_{1}$ from $5 \mathrm{~mm}$ to $80 \mathrm{~mm}$ and for a bulk liquid temperature of $1.8 \mathrm{~K}$ to $2.14 \mathrm{~K}$ at $101.3 \mathrm{kPa}$.

\section{EXPERIMENTAL RESULTS}

\section{A. Steady-State Critical Heat Flux}

The experimental data of steady-state critical heat fluxes (CHF's), $q_{c r}$, for $50 \%$ and $9 \%$ orifices are shown versus bulk liquid temperature in Fig. 2 with a distance between the flat plate heater and the orifice, $L_{1}$, as parameter. As is shown in the figure, $q_{c r}$ depends little on $L_{1}$. The lower the bulk liquid temperature becomes, the higher the CHF for each $L_{1}$ is. The critical heat flux, $q_{c r}$, for $50 \%$ orifice is higher than that for $9 \%$ orifice at each liquid temperature and the ratio of the former to the latter becomes higher with the decrease in the liquid bath. 


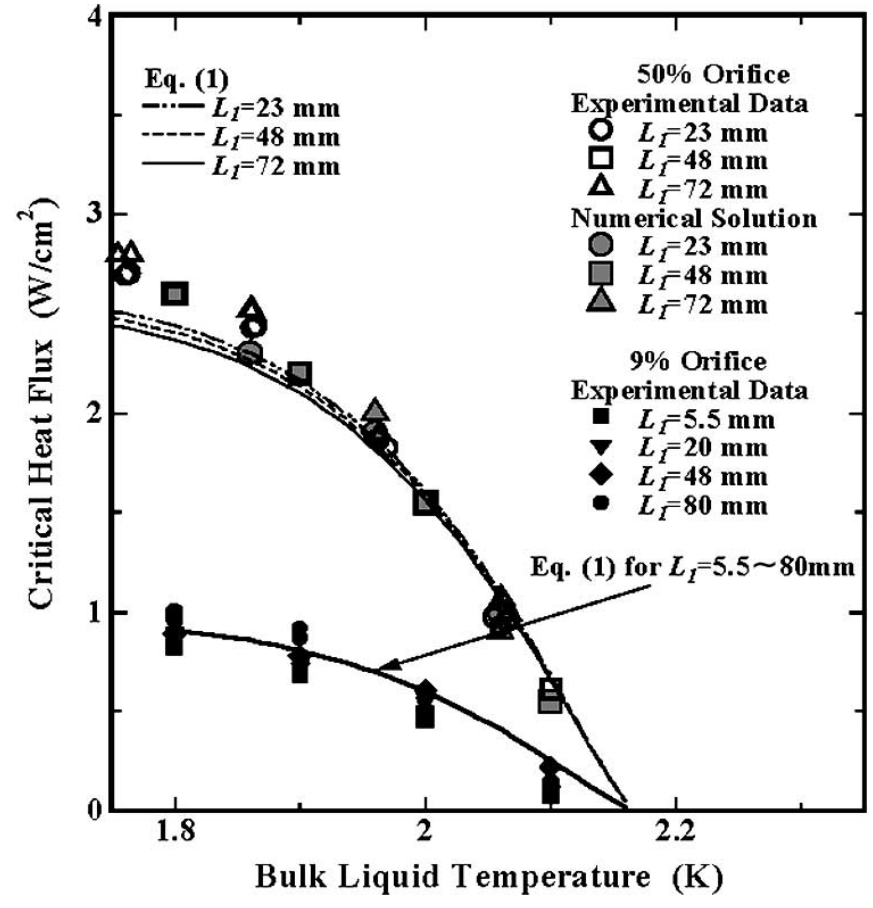

Fig. 2. Relationship between CHF and bath liquid temperature.

\section{B. Correlation of $\mathrm{CHF}$}

Tatsumoto et al. [4] have derived the following correlation of $\mathrm{CHF}$ on a flat plate heater at one end of a rectangular duct with an orifice.

$$
q_{c r}=\frac{1}{A_{1}\left\{\frac{L_{1}}{A_{1}^{3}}+\frac{L_{2}}{A_{2}^{3}}+\frac{\left(\frac{1}{k^{\prime}}\right)}{A_{2}^{3}}\right\}^{\frac{1}{3}}}\left(\int_{T_{B}}^{T_{\lambda}} f(T)^{-1} d T\right)^{\frac{1}{3}}
$$

where

$\left.\frac{1}{k^{\prime}}=\left[\left(\frac{L_{3} A_{2}^{3}}{A_{3}^{3}}\right)^{\frac{3}{2}}+\left(\frac{w}{0.78}\right)^{\frac{3}{2}}\right) \cdot \tanh \left\{5\left(\frac{A_{3}}{A_{2}}-1\right)\right\}\right]^{\frac{2}{3}}$,

and $f(T)^{-1}$ is effective thermal conductivity function, $L_{1}$ and $A_{1}$ are the length and the area of the first duct, $L_{2}$ and $A_{2}$ are those of the orifice, and $L_{3}$ and $A_{3}$ are those of the second duct. In Fig. 2, the curves predicted by (1) are also shown for comparison. Most of the predicted values agree with the experimental data within $15 \%$ difference. However, the data for $50 \%$ orifice at $L_{1}=72 \mathrm{~mm}$ are about $20 \%$ higher than the predicted values. This is assumed to be due to three-dimensional expansion of heat flow through orifice, as the orifice is placed very near to the end of the duct.

\section{Transient Heat Transfer Caused by a Stepwise Heat Input}

Typical behavior of transient heat transfer for stepwise heat input with a magnitude larger than $q_{c r}$ is as follows. Initially, the heat input rapidly increases and then it takes a constant value, $Q_{s}\left(\mathrm{~W} / \mathrm{m}^{3}\right)$, after $t=t_{A}$. The surface temperature difference and the heat flux remain constant at $\Delta T_{s}$ and $Q_{s}$ respectively, for a certain duration $\left(t_{B}-t_{A}\right)$, then they begin to increase and decrease, respectively, at a time $t=t_{B}$. The duration $t_{L}=$

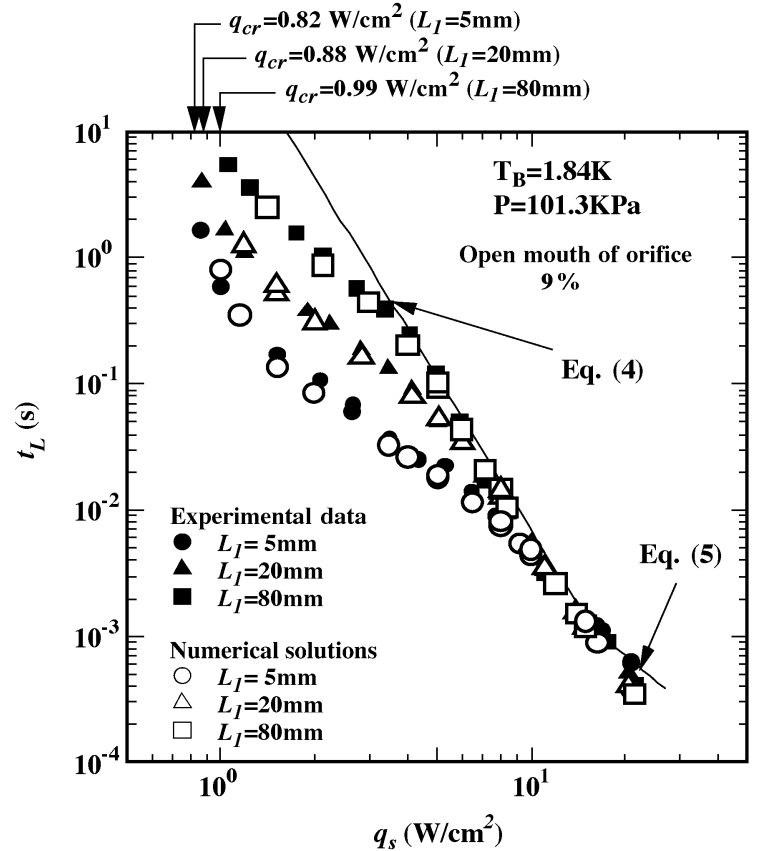

Fig. 3. Relationship between lifetime and bulk liquid temperature.

$t_{B}-t_{A}$ is defined as the lifetime of the quasi steady-state heat flux $q_{s}$.

The data of lifetime, $t_{L}$, for the duct with the $9 \%$ orifice for various stepwise heat flux, $q_{s}$ are displayed in Fig. 3 with the distance $L_{1}$ as a parameter. Shiotsu et al. [5] have presented the following correlation of lifetime for one-dimensional heat flow:

$$
\begin{aligned}
t_{L} & =a^{-4} \overline{\rho C_{p}} \overline{f(T)^{-1}}\left(T_{\lambda}-T_{B}\right)^{2} q_{s}^{-4} \\
t_{L} & =\overline{\rho C_{p}} \overline{B(T)^{-1}}\left(T_{\lambda}-T_{B}\right)^{2} q_{s}^{-2}
\end{aligned}
$$

where $a=1.16, B(T)^{-1}=s^{3} T / A^{*}, A^{*}=8000 \mathrm{~m}^{3} /(\mathrm{kg} \cdot \mathrm{s})$, the $\rho$ is the density of He II, and the $C_{P}$ is the specific heat,

$$
\overline{\rho C_{p}}, \overline{f(T)^{-1}} \text { and } \overline{B(T)^{-1}}
$$

are those averaged over $T_{B}$ to $T_{\lambda}$.

The lifetime is infinite for $q_{s}<q_{c r}$. With increase in $q_{s}$ from $q_{c r}$, the lifetime rapidly decreases and becomes lower than the straight line with the gradient of -4 given by (4), and then approaches the line. The CHF's for various $L_{1}$ are almost constant as described above, and the lifetime for each $L_{1}$ approaches the same line. However, the lifetime for smaller $q_{s}$ is shorter for shorter $L_{1}$. It reaches the line given by (4) at $q_{s}$ of around 3 , 5 and $8 \mathrm{~W} / \mathrm{cm}^{2}$ for $L_{1}$ of $80,20,5 \mathrm{~mm}$, respectively. Then it decreases along the lines for the one-dimensional heat transfer given by (4) and (5).

\section{NUMERICAL ANALYSIS}

\section{A. Numerical Model}

In the numerical analysis, a two-dimensional model shown in Fig. 4 was applied to the system of a rectangular duct with an orifice.

The steady-state and transient heat transfer were analyzed with a numerical code named SUPER-2D developed by Tatsumoto et al. [2] based on the two-fluid model and the theory of the mutual friction. Two-fluid equations used in the analysis 


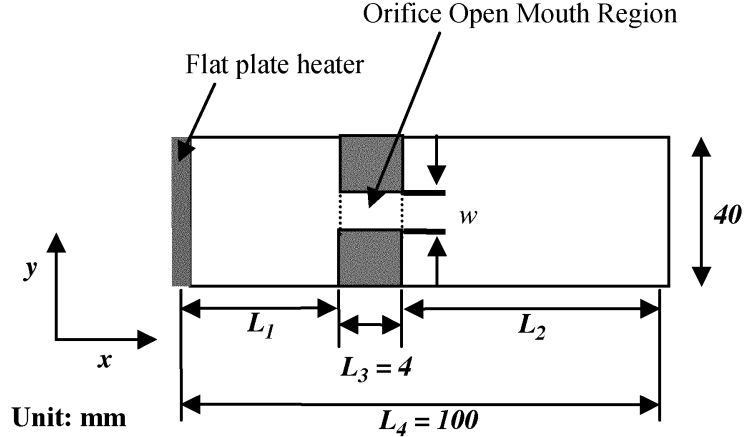

Fig. 4. Schematic of two-dimensional model of the system.

is expressed as follows. Details of the numerical code has been described elsewhere [2]:

- Momentum density

$$
\rho_{n} \boldsymbol{v}_{\boldsymbol{n}}+\rho_{s} \boldsymbol{v}_{\boldsymbol{s}}=\rho \boldsymbol{v}
$$

- Continuity equation for the total fluid

$$
\frac{\partial \rho}{\partial t}+\nabla \cdot\left(\rho_{n} \boldsymbol{v}_{\boldsymbol{n}}+\rho_{s} \boldsymbol{v}_{\boldsymbol{s}}\right)=0
$$

- Momentum equation for the total fluid

$$
\begin{aligned}
\frac{\partial\left(\rho_{n} \boldsymbol{v}_{\boldsymbol{n}}+\rho_{s} \boldsymbol{v}_{\boldsymbol{s}}\right)}{\partial t}= & -\nabla:\left(\rho_{n} \boldsymbol{v}_{\boldsymbol{n}} \boldsymbol{v}_{\boldsymbol{n}}+\rho_{s} \boldsymbol{v}_{\boldsymbol{s}} \boldsymbol{v}_{\boldsymbol{s}}\right)-\nabla P \\
& +\eta\left(\nabla^{2} \boldsymbol{v}_{\boldsymbol{n}}+\left(\frac{1}{3}\right) \nabla\left(\nabla \cdot \boldsymbol{v}_{\boldsymbol{n}}\right)\right)+\rho g
\end{aligned}
$$

- Momentum equation of the superfluid component

$$
\begin{aligned}
\frac{\partial \boldsymbol{v}_{\boldsymbol{s}}}{\partial t}+\boldsymbol{v}_{\boldsymbol{s}} \cdot \nabla \boldsymbol{v}_{\boldsymbol{s}}=- & \left(\frac{1}{\rho}\right) \nabla P+A \rho_{n}\left|\boldsymbol{v}_{\boldsymbol{n}}-\boldsymbol{v}_{\boldsymbol{s}}\right|^{2}\left(\boldsymbol{v}_{\boldsymbol{n}}-\boldsymbol{v}_{\boldsymbol{s}}\right) \\
& +\left(\frac{\rho_{n}}{2 \rho}\right) \nabla\left|\boldsymbol{v}_{\boldsymbol{n}}-\boldsymbol{v}_{\boldsymbol{s}}\right|^{2}+S \nabla T+g
\end{aligned}
$$

\section{- Entropy equation}

$$
\frac{\partial(\rho S)}{\partial t}+\nabla \cdot\left(\rho S \boldsymbol{v}_{\boldsymbol{n}}\right)=\frac{A \rho_{n} \rho_{s}\left|\boldsymbol{v}_{\boldsymbol{n}}-\boldsymbol{v}_{\boldsymbol{s}}\right|^{4}}{T}
$$

where $v$ is the velocity, $T$ is the temperature, $P$ is the pressure, t is the time, $\eta$ is the viscosity, $S$ is the specific entropy, $\rho$ is the density, and $A$ is the Gorter-Mellink mutual friction parameter. The subscripts, $s$ and $n$, denote the superfluid and the normal fluid components, respectively.

The two-fluid equations were solved by finite difference method with the staggered grid sized $0.25 \mathrm{~mm} \times 0.25 \mathrm{~mm}$. Time integration was performed explicitly with a time step of $4.0 \times 10^{-7}$. The adiabatic condition is applied to the duct wall except the heated section. The temperature at the channel end is kept constant at bath temperature. The liquid temperature on the heated surface is calculated by solving the form for a one-dimensional transient heat transfer with iteration [6]:

$$
\rho C_{P}\left(\frac{\partial T}{\partial t}\right)=\frac{\partial\left(\frac{f(T)^{-1} \partial T}{\partial y}\right)^{\frac{1}{3}}}{\partial y} .
$$

The full slip condition is applied to velocities of the super-fluid component in the direction tangential to the wall, and velocities
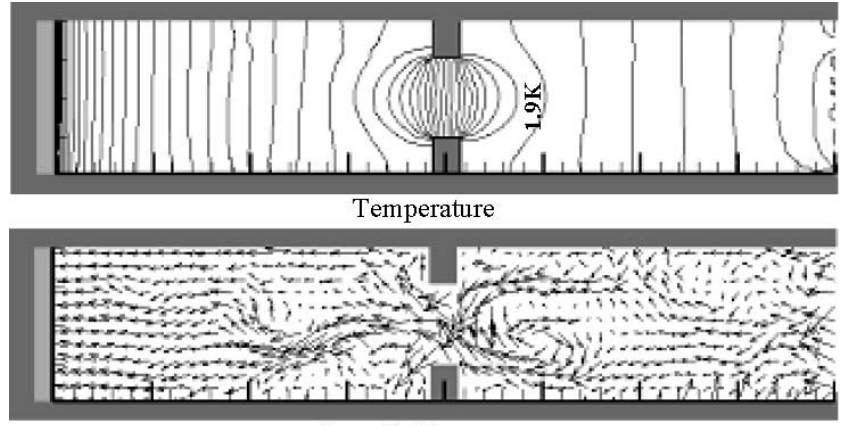

Superfluid component

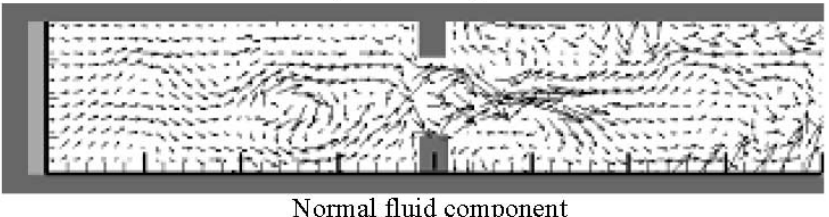

Normal fluid component

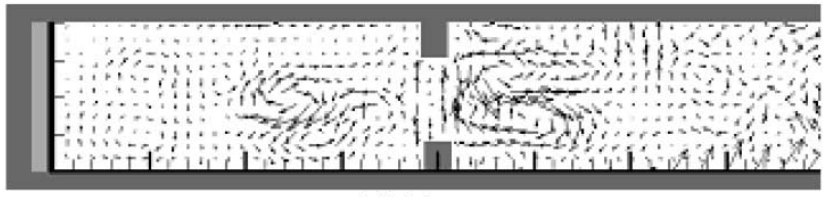

Total fluid component

Fig. 5. The velocity of each component and the temperature distribution for $L 1=48 \mathrm{~mm}$ under steady-state critical heat flux condition. Temperature gap between the isothermal lines is $0.005 \mathrm{~K}$ and the vector of the length of $1 \mathrm{~mm}$ in this graphic is equivalent to the flow velocity of $30 \mathrm{~cm} / \mathrm{sec}$.

of the normal fluid component on the wall are set to be zero. The velocities of both super and normal fluid components at the heated surface in the direction perpendicular to the surface are expressed as follows:

$$
\boldsymbol{v}_{\boldsymbol{n}}=\frac{q}{(\rho S T)}, \quad \boldsymbol{v}_{\boldsymbol{s}}=\frac{-\rho_{n} q}{\left(\rho_{s} \rho S\right) T}
$$

In case of steady-state heat transfer analysis, when $(\partial T / \partial t)$ at the heated surface converged, the temperature distribution was regarded to be steady state. The heat flux applied to the heater was set to be $0.04 \mathrm{~W} / \mathrm{cm}^{2}$ higher than the previous value and the calculation was repeated. And when the temperature reached $T_{\lambda}$, the heat flux was defined as the steady-state critical heat flux, $q_{c r}$. In case of transient heat transfer analysis, the heat flux was kept constant until the $\lambda$ transition occurred.

\section{NUMERICAL RESULTS}

\section{A. Steady-State Critical Heat Flux}

The solutions of CHF for 50\% orifice are shown in Fig. 2 in terms of solid symbols. The solutions of $\mathrm{CHF}$ given by the analysis match the experimental data within $10 \%$ difference. The solutions of CHF show that CHF for various bulk liquid temperature depend little on the orifice locations.

The velocity of each component and the temperature distribution for $L_{1}=48 \mathrm{~mm}$ under steady-state critical heat flux condition is displayed in Fig. 5. These plots make us realize that the vortices caused by the orifice exist near the entrance and the exit of the orifice in each velocity vector distribution. It is supposed that these vortices cause the two-dimensional temperature distribution around the orifice. 

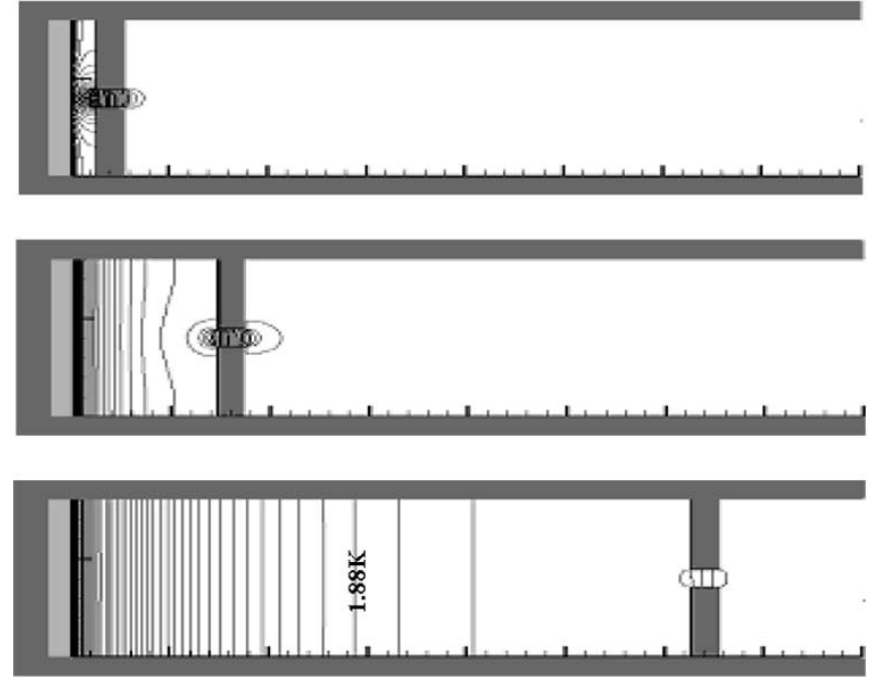

Fig. 6. Temperature distribution at $q=6.5 \mathrm{~W}$ for $L_{1}=5,20,80 \mathrm{~mm}$ described with a temperature gap between the isothermal lines $0.005 \mathrm{~K}$.

\section{B. Transient Heat Transfer}

The solutions of lifetime are shown in Fig. 3 in terms of open symbols. The solutions for each $L_{1}$ agree well with the experimental data. For $q_{s} \geq 6.5 \mathrm{~W} / \mathrm{cm}^{2}$, the solutions on each orifice location almost fit the line given by (4). For $q_{s} \leq 6.5 \mathrm{~W} / \mathrm{cm}^{2}$, the solutions are lower than the line given by (4). The closer to the flat plate heater the orifice location is, the larger the difference between the solution and the line becomes.

Fig. 6 shows the temperature distribution in the duct with a step heat flux $q_{s}$ of $6.5 \mathrm{~W} / \mathrm{cm}^{2}$ for various $L_{1}$ of $5,20,80 \mathrm{~mm}$. The temperature distribution for $L_{1}=5 \mathrm{~mm}$ shows that the heat transfer depends not only on the upstream duct but on the area of the orifice and that a significantly narrow temperature distribution was formed in the orifice open mouth region. The temperature distribution for $L_{1}=20 \mathrm{~mm}$ also shows the similar effect of the orifice as the temperature distribution for $L_{1}=5 \mathrm{~mm}$ does, although the effect is less significant. The temperature distribution or $L_{1}=80 \mathrm{~mm}$ shows the effect of the orifice is quite small.

\section{CONCLUSION}

Heat transfer in a rectangular duct with an orifice was investigated experimentally and numerically.

The experimental CHF's are lower for smaller orifice open mouth and higher bulk liquid temperature and depend little on the orifice location. Numerical analysis can describe these experimental data within $10 \%$ difference. It is clear from the analysis that the vortices are formed near the entrance and exit of the orifice. The vortices disturb the heat transfer.

The solutions of transient heat transfer show that the lifetime depends significantly on the orifice location whereas CHF's depend little on the location. The lifetime for smaller heat input is shorter for shorter distance between orifice and flat plate heater. It is shown that the numerical solutions of transient heat transfer lifetime agree well with the experimental data. According to the numerical analysis, it can be understood that temperature distribution with steep gradient is generated across the orifice when the orifice is closer to the flat plate heater.

\section{REFERENCES}

[1] J. M. Pfotenhauer, "The design of stable race-track superconducting switch," Cryogenics, vol. 32, p. 466, 1992.

[2] H. Tatsumoto, K. Fukuda, and M. Shiotsu, "Numerical analysis for steady-state two-dimensional heat transfer from a flat plate at one side of a duct containing pressurized He II," Cryogenics, vol. 42, pp. 9-28, 2002.

[3] H. Tatsumoto, K. Hama, K. Hata, M. Shiotsu, and Y. Shirai, "Heat transfer on a flat plate at one end of a series-connected rectangular duct in pressurized He II," IEEE Trans. Appl. Supercond., vol. 12, no. 1, pp. 1364-1367, March 2002.

[4] H. Tatsumoto, K. Hata, K. Hama, Y. Shirai, and M. Shiotsu et al., "Critical heat fluxes on a flat plate pasted on one end of a rectangular duct with an orifice in pressurized He II," in Advances in Cryogenic Engineering, S. Breon et al., Eds: American Institute of Physics, 2002, vol. 47B, pp. 1364-1371.

[5] M. Shiotsu, K. Hata, Y. Takeuchi, K. Hama, and Y. Shirai et al., "Transient heat transfer caused by a stepwise heat input to a flat plate in pressurized He II," in Proceedings of International Cryogenic Engineering Conference 1998, D. Dew-Hughes et al., Eds. Bristol: Institute of Physics Publishing, 1998, pp. 687-690.

[6] S. W. Van Sciver, Helium Cryogenics. New York: Plenum Press, 1986. 